\title{
Accuracy of Quantum Sensors Measuring Yield Photon Flux and Photosynthetic Photon Flux
}

\author{
Charles Barnes', Theodore Tibbitts', John Sager ${ }^{3}$, Gerald Deitzer', \\ David Bubenheim ${ }^{5}$, Gus Koerner, and Bruce Bugbee \\ Department of Plants, Soils, and Biometeorology, Utah State University, \\ Logan, UT 84322-4820 \\ Additional index words. controlled environments, electric lamps, growth chambers, light \\ quality, radiation, spectral photon distribution
}

\begin{abstract}
Photosynthesis is fundamentally driven by photon flux rather than energy flux, but not all absorbed photons yield equal amounts of photosynthesis. Thus, two measures of photosynthetically active radiation have emerged: photosynthetic photon flux (PPF), which values all photons from 400 to $700 \mathrm{~nm}$ equally, and yield photon flux (YPF), which weights photons in the range from 360 to $760 \mathrm{~nm}$ according to plant photosynthetic response. We selected seven common radiation sources and measured YPF and PPF from each source with a spectroradiometer. We then compared these measurements with measurements from three quantum sensors designed to measure YPF, and from six quantum sensors designed to measure PPF. There were few differences among sensors within a group (usually $<5 \%$ ), but YPF values from sensors were consistently lower (3\% to $20 \%$ ) than YPF values calculated from spectroradiometric measurements. Quantum sensor measurements of PPF also were consistently lower than PPF values calculated from spectroradiometric measurements, but the differences were $<7 \%$ for all sources, except red-light-emitting diodes. The sensors were most accurate for broad-band sources and least accurate for narrow-band sources. According to spectroradiometric measurement, YPF sensors were significantly less accurate ( $>9 \%$ difference) than PPF sensors under metal halide, high-pressure sodium, and low-pressure sodium lamps. Both sensor types were inaccurate ( $>18 \%$ error) under red-light-emitting diodes. Because both YPF and PPF sensors are imperfect integrators, and because spectroradiometers can measure photosynthetically active radiation much more accurately, researchers should consider developing calibration factors from spectroradiometric data for some specific radiation sources to improve the accuracy of integrating sensors.
\end{abstract}

Accurate measurement of photosynthetic radiation is essential for controlled-environment research, particularly when comparisons

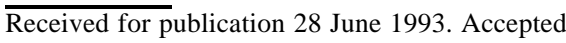
for publication 6 July 1993. This research was supported by NASA Cooperative Agreement no. NCC2-139; the Utah Agricultural Experiment Station;andtheBiotron, Univ. of Wisconsin, Madison. Approved as UAES journal paper no. 4197. Mention of trade names does not constitute an endorsement by the authors, We appreciate the helpful review comments from personnel at Skye Instruments and LI-COR, and from Cary Mitchell. Skye InstrumentsmanufacturesaPPF sensor with identicalspecifications and a similar spectral response to theLI-CORPPFsensor, but, unfortunately, we were notabletoinclude the Skye PPF sensor in this study, The cost of publishing this paper was defrayed in part by the payment of page charges. Under postal regulations, this paper therefore must be hereby marked advertisement solely to indicate this fact.

'Assistant Professor, Northland College, Ashland, WI 54806.

${ }^{2}$ Dept. of Horticulture, Univ. of Wisconsin, Madison, WI 53706.

${ }^{3}$ NASA, Mail Code MD-RES, John F. Kennedy Space Center, FL 32899.

${ }^{4}$ Dept. of Horticulture, Univ. of Maryland, College Park, MD 20742.

'NASA Ames Research Center, MS 239-4, Moffett Field, CA 94035. are made among electric lamps with different spectral emission characteristics. The criteria and methods used for measuring photosynthetically active radiation (PAR; the wavelengths that drive photosynthesis) have been investigated intensively over the past two decades (McCree, 1972a, 1972b, 1981; McPherson, 1969; Sager et al., 1982, 1988; Tibbitts et al., 1986). The most common method of measuring PAR gives equal value to all photons with wavelengths between 400 and $700 \mathrm{~nm}$ and is referred to as the photosynthetic photon flux (PPF), measured in units of moles per square meter per second. The ideal PPF sensor would respond equally to all photons between 400 and 700 nm (McPherson, 1969; Tibbitts et al., 1986) (Fig. la). However, photosynthesis is driven by photons with wavelengths below $400 \mathrm{~nm}$ and above $700 \mathrm{~nm}$, and photons of different wavelengths induce unequal amounts of photosynthesis (Inada, 1976, 1978a; McCree, 1972a, 1972b, 1981). For these reasons, an accurate measurement of PAR should follow the relative quantum efficiency (RQE) curve (Fig. lb) originally developed by McCree (1972a), which weights the photosynthetic value of all photons with wavelengths from 360 to $760 \mathrm{~nm}$. A sensor that responds according to this curve measures yield photon flux (YPF) in moles per square meter per second, the same units as for PPF.

The Stark-Einstein Law states that one absorbed photon excites one electron regardless of the photon's energy between 400 and $700 \mathrm{~nm}$; this law is the basis for weighting photons equally. However, although $>90 \%$ of blue photons are absorbed, $\approx 20 \%$ of these photons are absorbed by inactive pigments; their energy is not transferred to energy-collecting pigments (reaction centers) and is lost as heat and fluorescence. This loss means that the quantum yield of absorbed blue photons is typically $\approx 20 \%$ less than the quantum yield of absorbed red photons. Species differ in their proportion of inactive pigments. Inada (1977) found that both radiation absorption and quantum yield in the middle wavelengths (500-600 $\mathrm{nm}$ ) were substantially lower in purple, fieldgrown leaves than in green ones because of inactive absorption by anthocyanin. Clark and Lister (1975) found that blue and blue-green Colorado spruce species had high concentrations of inactive carotenoids, which reduced blue light (400-500 run) absorption and quantum yield. Environment can also influence the concentration of inactive pigments. McCree (1972a) found that growth-chamber-grown plants tended to use ultraviolet and blue radiation more efficiently than field-grown plants, and this may be due to a lower concentration of inactive pigments in nonstressed growthchamber-grown plants.

In spite of these genetic and environmental influences on quantum yield, McCree (1972a) found that the spectral quantum yield of healthy, green leaves of 22 crop plant species differed by less than $\pm 5 \%$, so he defined an average YPF curve. Inada ( 1976) obtained a second set of comprehensive quantum yield data (from 33 species) and confirmed McCree's (1972a) measurements.

Quantum sensors designed to measureYPF or PPF are commercially available. Both types use multiple-spectral filters in front of a broadspectrum radiation detector, but neither type matches its desired curve (Fig. $1 \mathrm{a}$ and b). Our objective was to determine the accuracy of each type of sensor for solar radiation and for six electric lamps. Measurements from each sensor were compared with YPF and PPF values calculated from measurements made with a spectroradiometer.

We tested three YPF quantum sensors (model SKP-21O; Skye Instruments, Liandrindod Wells, Powys, England) designed to measure PAR from $\approx 340$ to $760 \mathrm{~nm}$ (Fig. lb). The Skye sensors were previously unused and had been manufactured within the previous 9 months. The three Skye sensors deviated from each other by $<5 \%$ under all sources. None of these was consistently high or low, so data are reported as the means of the three sensors. PPF was measured using a LI-COR quantum sensor (model LI-190SB; LI-COR, Lincoln, Neb.) calibrated by the manufacturer within the previous year. The quantum sensor was returned to the manufacturer after the study to verify that the calibration had not changed. This sensor was later compared with five other recently calibrated LI-COR quantum sensors tested under the same, seven light sources. 


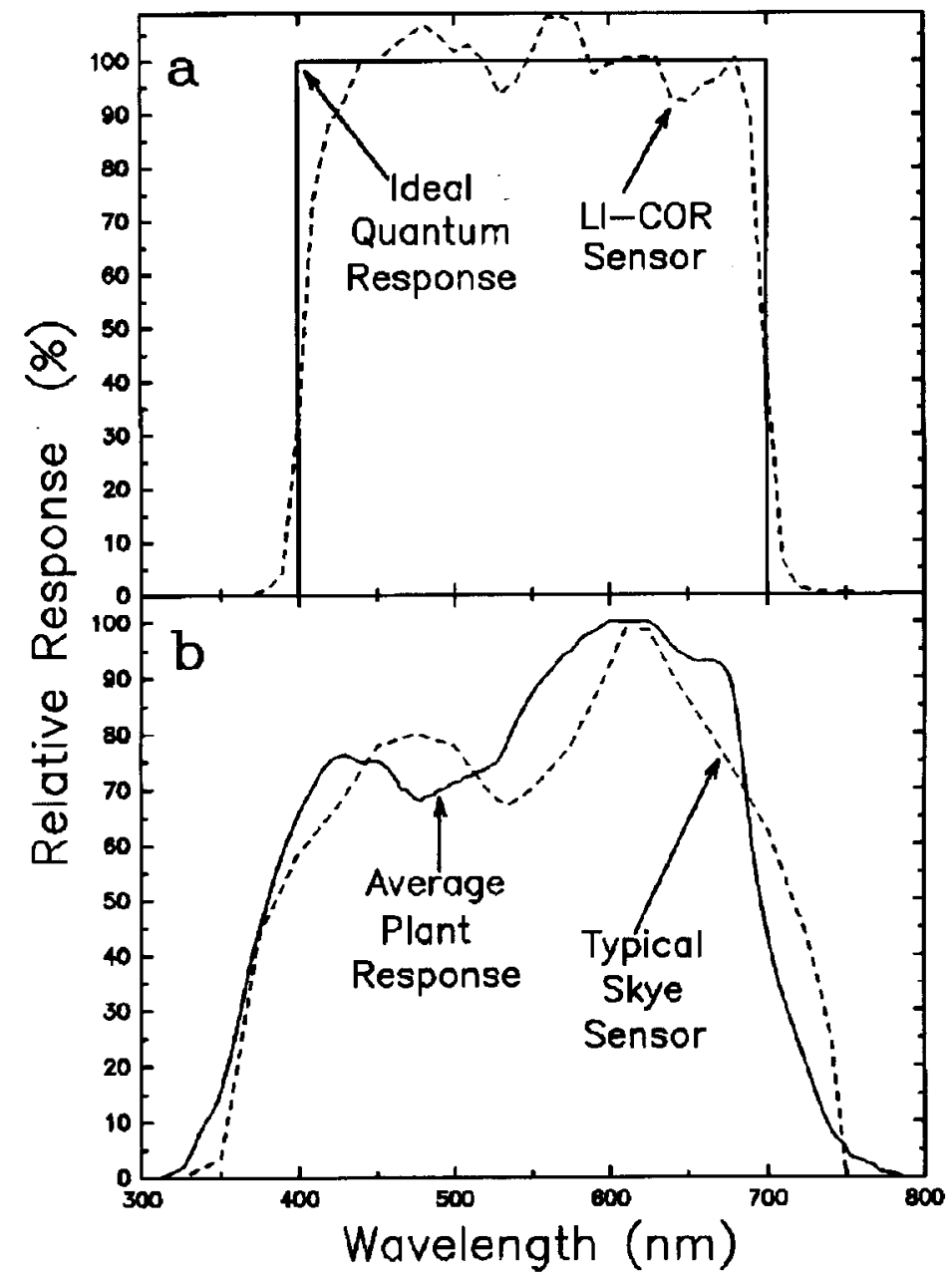

Fig. 1. (a) A comparison of the ideal quantum photosynthetic photon flux response with the actual response of the LI-COR sensor used in this study. The response curve of the LI-COR sensor is typical of LI-COR sensors in general (based on data supplied by manufacturer). The ideal response shown here differs from the response shown in LI-COR literature because LI-COR shows the amount of filtering that must occur to achieve the flat response curve, rather than the flat response curve itself. Because the LI-COR detector (in the base of the quantum sensor) fundamentally measures energy, high-energy blue photons must be filtered more than red photons so that the sensor responds equally to all photons. It is much less confusing to show directly the ideal, equal photon response rather than the amount of filtering to achieve the response. (b) A comparison of the relative quantum efficiency curve as determined by the average plant response for photosynthesis (McCree, 1972a), with the actual response curve of a typical Skye sensor (based on data supplied by the manufacturer).
Measurements among the LI-COR quantum sensors also were uniform and varied $<5 \%$ under all sources, except measurements for red-light-emitting diodes (RLEDs), which varied as much as $10 \%$. RLED measurements varied more among individual sensors within each group than did any other source. This variation probably occurred because peak output from the RLEDs $(660 \mathrm{~nm})$ is near the far-red filter cutoff for both types of sensors (Fig. $1 \mathrm{a}$ and $\mathrm{b}$ ), and the exact cutoff wavelength varies slightly with different batches of filters in the sensors [Larry Middendorf ments), personal communication]. Spectral photon distribution (SPD) files in energy flux (watts per square meter per second per nanometer) and photon flux units (moles per square meter per second per nanometer) were generated either by a LI-COR (model LI-1800) or an Optronics (model 740A; Orlando, Fla.) spectroradiometer. The spectral emission char(LI-COR) and Belinda Trotter (Skye Instru- lated by multiplying the SPD files by the RQE values of Sager et al. (1988), which are based on average values reported by McCree (1972a). PPF was calculated from the SPD files by summing the photon flux over the 400- to 700$\mathrm{nm}$ interval. Both spectroradiometers were calibrated against National Institute of Standards and Technology standards and compared to each other under common sources to check uniformity. All measurements were taken at precisely the same distance and angle from each of the seven sources. We found, however, that the output of all the lamps can vary by about $\pm 2 \%$ over short time intervals (because of changing temperature and line voltage), and it is difficult to reproduce exactly spectroradiometer measurements, so differences $<5 \%$ are not significant.

A comparison with spectroradiometric measurements shows the Skye sensors undersources tested (Table 1). The Skye sensors performed best under broad-spectrum sources, including solar, incandescent, and cool-white fluorescent lamps, where measured values were $<10 \%$ lower than spectroradiometric values. The values obtained with the Skye sensors were considerably lower than spectroradiometric values under narrow-band sources or sources having a large fraction of their output at discrete wavelengths, i.e., metal halide (MH), high-pressure sodium (HPS), and low-pressure sodium (LPS) lamps, and RLEDs. The Skye sensors were not recalibrated after the study, but the three sensors were uniform, so if the output had changed in the 9 months since manufacture, the change was identical in all sensors. The uniformity of the Skye sensors made large calibration changes extremely unlikely, but the output from quantum sensors tends to drift downward by $» 1 \%$ per year (Belinda Trotter and Larry Middendorf, personal communication), so a small decrease in output could have occurred for all three sensors.

Similarly, the LI-COR quantum sensor underestimated PPF for each of the seven sources tested (Table 1); however, most of the errors were within the measurement accuracy of the spectroradiometer (5\%). For three sources (MH, HPS, and LPS), the error assoestimated YPF for each of the seven radiation acteristics of the sources are energy flux (Fig 2a) and photon flux (Fig. 2b). YPF was calcu-
Table 1. Comparison of yield photon flux (YPF) as measured by three Skye sensors and a spectroradiometer, and of photosynthetic photon flux (PPF) as measured by aLI-COR quantum sensor and a spectroradiometer. Values for the Skye sensors represent the mean of the three sensors tested. Measurements are listed in micromoles per square meter per second; all values were repeatable within $\pm 3 \%$. The difference between the sensors' measurements of YPF or PPF and from the comparable measurement from the spectroradiometer are listed as the percent difference.

\begin{tabular}{|c|c|c|c|c|c|c|c|}
\hline \multirow[b]{2}{*}{$\begin{array}{l}\text { Radiation } \\
\text { source }^{z}\end{array}$} & \multicolumn{3}{|c|}{ YPF } & \multicolumn{3}{|c|}{ PPF } & \multirow[b]{2}{*}{$\begin{array}{c}\text { PPF : YPF } \\
\text { ratio }\end{array}$} \\
\hline & Skye & $\begin{array}{c}\text { Spectro- } \\
\text { radiometer }\end{array}$ & $\begin{array}{c}\text { Difference } \\
(\%)\end{array}$ & LI-COR & $\begin{array}{c}\text { Spectro- } \\
\text { radiometer }\end{array}$ & $\begin{array}{c}\text { Difference } \\
(\%)\end{array}$ & \\
\hline Solar & 1648 & 1701 & -3 & 1867 & 1924 & -3 & 1.13 \\
\hline INC & 121 & 126 & -4 & 124 & 132 & -6 & 1.05 \\
\hline CWF & 17.2 & 18.3 & -6 & 19.9 & 20.5 & -3 & 1.12 \\
\hline MH & 85 & 98 & -13 & 104 & 109 & -4 & 1.11 \\
\hline HPS & 144 & 175 & -18 & 171 & 183 & -7 & 1.05 \\
\hline LPS & 108 & 134 & -19 & 130 & 136 & -4 & 1.01 \\
\hline RLED & 64 & 80 & -20 & 74 & 90 & -18 & 1.12 \\
\hline
\end{tabular}

${ }^{2}$ Solar = sunlight; $\mathrm{INC}=$ incandescent CWF $=$ cool-white fluorescent; $\mathrm{MH}=$ metal halide; HPS $=$ high pressure sodium; LPS $=$ low-pressure sodium; RLED = red-light-emitting diodes.

'From spectroradiometer. 

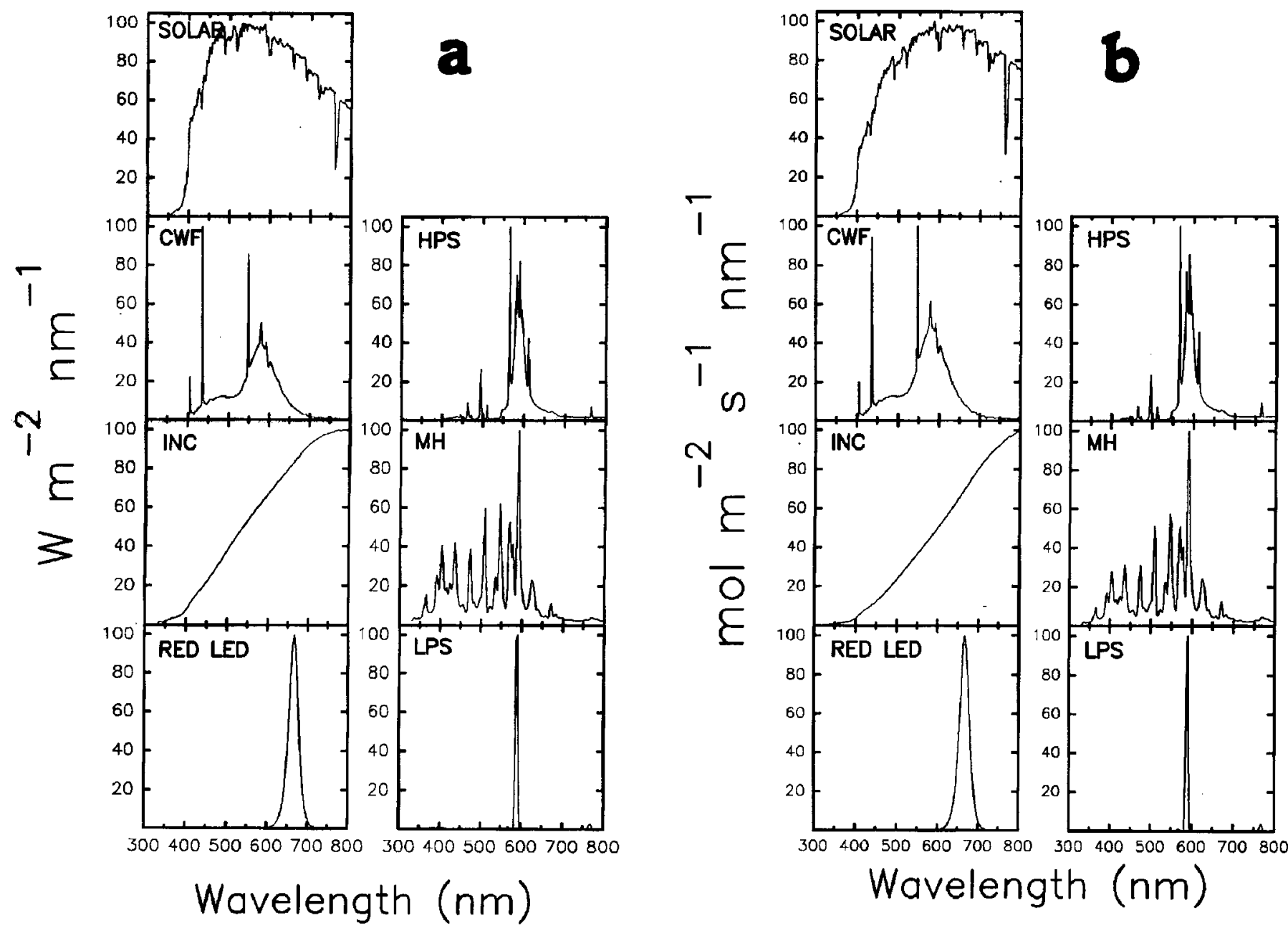

Fig. 2. The spectral characteristics of the seven radiation sources examined in this study. Data are normalized to a peak value of $100 \mathrm{~W} \cdot \mathrm{m}^{-2} \cdot \mathrm{nm} \mathrm{m}^{-1}$ to facilitate comparison among lamp types. (a) Data plotted on an energy flux basis, which is the most common method of expressing spectral distribution. (b) The same dataexpressedonaphoton-flux basis, which is a better predictor of plant response than energy flux. The difference is insignificant for monochromatic sources, but the relative peak heights change in broad-band sources. The solar curve drops off much less rapidly when expressed on a photon-flux basis than when expressedonanenergy basis. The solar curve was measured on a sunny summer day, and the shape of the curve depends on the ratio of direct to diffuse radiation and, thus, sun angle (Monteith and Unsworth, 1990). Increasing diffuse radiation shifts the peak to shorter wavelengths.

ciated with Skye sensors was 3 to 5 times greater than that associated with the LI-COR PPF sensor.

Spectroradiometric measurements of PPF exceeded YPF for every radiation source, and the difference was $>10 \%$ for four of the seven sources (Table 1). Sager et al. (1988) reported that PPF exceeded YPF by $25 \%$ for radiation sources with a strong blue component (400 to 500 rim). The differences for radiation sources with strong blue emissions (solar, $\mathrm{MH}$, and cool-white fluorescent) occurs because the YPF weighting factors are $<0.75$ for wavelengths $<530 \mathrm{~nm}$. PPF and YPF values are similar for lamps with peak outputs in the yellow-red region, because the YPF weighting factors are close to 1.0 for wavelengths around $600 \mathrm{~nm}$. PPF values were $12 \%$ higher than YPF values for RLEDs because the YPF curve drops off sharply at $>675 \mathrm{~nm}$.

Although there can be significant differences $(210 \%)$ between PPF and YPF, it has not been shown that photosynthesis and plant growth are more accurately predicted by the integrated YPF value than by the integrated
PPF value. The YPF curve was developed from measurements with monochromatic radiation, and it is well known that the quantum yield at some wavelengths can be increased by simultaneous irradiation at other wavelengths (Inada, 1978a). Both McCree (1972a) and Inada (1978b), however, found that "white" light negligibly enhanced the quantum yield in monochromatic radiation (summarized in McCree, 1981).

Thus, it maybe advantageous to determine YPF for some radiation sources, but for $\mathrm{MH}$, HPS, and LPS lamps, and for RLEDs, there were substantial errors with a quantum sensor designed to measure YPF. A PPF quantum sensor was relatively accurate in measuring PPF, but this may not provide the best estimate of PAR. Values obtained with either type of sensor should be compared to values for PPF or YPF that have been calculated from spectroradiometric measurements when measurements have been taken under narrow-band electric lamps or filtered radiation sources. A calibration factor can be derived to adjust values obtained from a particular source.
Our data did not indicate significant errors associated with LI-COR quantum sensors under the three most common radiation sources: sunlight, cool-white fluorescent, and $\mathrm{MH}$. The additional expense of making spectroradiometric measurements is not warranted for conventional studies under these radiation sources. This is particularly true since spectroradiometers are complex, calibrated instruments and are thus imperfect standards.

\section{Literature Cited}

Clark, J. and G. Lister. 1975. Photosynthetic action spectraof trees: I: Comparative photosynthetic action spectra of one deciduous and four coniferoustree species as related to photorespiration and pigment complements. Plant Physiol. 55:401-406

Inada,K.1976.Actionspectraforphotosynthesisin higher plants. Plant Cell Physiol. 17:355-365.

Inada, K. 1977. Effects of leaf color and the light qualityappliedto leaf-developing period on the photosynthesis response spectra in crop plants. Proc. Crop Sci. Soc. Jpn. 46:37-44.

Inada, K. 1978a. Photosynthetic action spectra in 


\section{Miscellaneous}

higher plants. Plant Cell Physiol. 19:1007-1017. Inada, K. 1978b. Spectral dependence of photosynthesis in crop plants. Acts Hort. 87:177-184.

McCree, K.J. 1972a. The action spectrum, absorbance and quantum yield of photosynthesis in crop plants. Agr. Meteorol. 9:191-216.

McCree, K.J. 1972b. Test of current definitions of photosynthetically active radiation against leaf photosynthesis data. Agr. Meteorol. 1043453.

McCree, K.J. 1981. Photosynthetically active radiation, p. 41-55. In: O.L. Lange, P.S. Nobel, C.B.
Osmund, and H. Ziegler (eds.). Encyclopedia of plant physiology. New series, vol. 12A.Physiological plant ecology I. Springer Verlag, Berlin.

McPherson. H.G. 1969. Photocell-filter combinations for measuring photosynthetically active radiation. Agr. Meteorol. 6:347-356.

Monteith, J.L. and M.H. Unsworth. 1990. Principles of environmental physics. 2nd ed. Edward Arnold, London.

Sager, J. C., J.L. Fdwards, and W. H. Klein. 1982. Light energy utilization efficiency for photo- synthesis. Trans. Amer. Soc. Agr. Eng. 25(6):1737-1746.

Sager, J. C., W.O. Smith, J.L. Edwards, and K.L. Cyr. 1988. Photosynthetic efficiency and phytochrome photoeauilibria determination using spectral data. Trans. Amer. Soc. Agr. Eng. 31(6): 1882-1889.

Tlbbitts, T.W., D.A. McSparron, and D.T. Krizek. 1986. Spectral effects on the use of photon flux sensors for measurement of photosynthetic photon flux in controlled environments. Biotronics 15:31-36. 